\title{
Exploring metaphor's effects in reasoning on vaccination
}

\author{
Francesca Ervas $^{* 12}$, Pietro Salis ${ }^{1}$, Rachele Fanari ${ }^{1}$ \\ ${ }^{1}$ Department of Education, Psychology, Philosophy, University of Cagliari, Cagliari, Italy \\ ${ }^{2}$ Netherlands Institute for Advanced Study in the Humanities and Social Sciences, \\ Amsterdam, The Netherlands \\ * Corresponding author \\ Email: ervas@unica.it
}

\begin{abstract}
The paper investigates the impact of the use of metaphors in reasoning tasks concerning vaccination, especially for defeasible reasoning cases. We assumed that both metaphor and defeasible reasoning can be relevant to let people understand vaccination as an important collective health phenomenon, by anticipating possible defeating conditions. We hypothesized that extended metaphor could improve both the argumentative and the communicative effects of the message. We designed an empirical study to test our main hypotheses: participants were presented with a text about vaccination, described in either literal or metaphorical terms, based on uncertain vs. safe reasoning scenarios. The results of the study confirmed that defeasible reasoning is relevant for the communicative impact of a text and that an extended metaphor enhances the overall communicative effects of the message, in terms of understandability, persuasion, perceived safety and feeling of control over the health situation, collective trust in expertise and uptake of experts' advice. However, the results show that this effect is significantly nuanced by the type of defeasible reasoning, especially in the case of participant's trust in expertise and commitment to experts' advice.
\end{abstract}

\section{Keywords}

Vaccination, Uncertain reasoning, Metaphor, Collective immunity, Defeasible reasoning, Trust, Vaccine communication 


\section{Introduction}

In times of COVID-19 pandemic, the promotion of collective vaccination in institutional campaigns is of utmost importance. It is crucial for public health to make the vital need for collective vaccination as much clear as possible especially to hesitant people, as COVID-19 vaccination is on a voluntary basis in most countries. Vaccine hesitancy is indeed an important issue to be addressed in institutional communication, especially in the context of a decrease in vaccination coverage and control of vaccine-preventable diseases. Even though it might be non-determinant for the individuals' care decision and behavior [35], an inadequate institutional communication might contribute to vaccine hesitancy and negatively influence the collective vaccination uptake. This issue is even more delicate as "recent debates about the risks suggest that public trust in immunization programmes is fragile" [7, p. 9] and possibly influenced by "media logic" rather than "epidemiological logic", with consequent loss of trust in public health authorities [43; 47; 23]. For instance, Peretti-Watel and colleagues [43] studied the "Pandemic Public Health Paradox" during the first pandemic of the 21 st century (influenza A H1N1 in 2009), i.e. the unexpected low vaccination rates at the onset of the second, most fatal, epidemic wave. Interestingly, they showed that the vaccination rates trends followed the decline of media attention for the disease, which influenced public risk perceptions and behaviors long before the epidemic's peak. As pointed out by Biasio and colleagues [3, p. 2986], "communication based on valid and shared strategies, as well as on coherent behaviors, can modify the attitudes toward vaccinations, becoming one of the main components of the global strategy to oppose vaccine hesitancy".

Patients' understanding of vaccination as a fundamental preventive measure should be considered as a priority in institutional communication about vaccination. Metaphors might be useful pedagogical devices in vaccine communication to explain a health phenomenon, which otherwise might remain unintelligible to laypeople. Metaphor can indeed be a way to grasp an unknown/less known concept (the target) by using a better-known concept (the source) [30;8]. Metaphors in health communication have been widely studied, especially in the case of cancer, and sometimes also criticized to the point of proposing "a liberation from them" [60, p. 4] as violent uses of language for people suffering from cancer treatments. A variety of metaphors have been proposed also in vaccine communication, ranging from the conventional military metaphor of the "garrison" to the novel metaphor of the "beehive" to let people understand the concept of "herd immunity" without making people feel mindless sheeps following the flock $[5 ; 15]$. Each metaphor provides a specific perspective to interpret both the disease and its 
development, as it entails a framing effect on the health phenomenon to be explained [56; 56]. However, it is not clear whether and which metaphorical frames have more chances to be successful in vaccine communication to achieve population's compliance in the management of vaccine-preventable diseases. Indeed, in the case of vaccination, we may even wonder whether metaphors are effective devices for patients' education or whether literal communication on vaccines and vaccine-preventable diseases might be preferable to implicit metaphorical communicated content.

Exposure to metaphorical framing proved capable to modify how people reason about a specific social problem [66; 67] (but see [64] for criticism), also in the case of metaphors used in vaccine communication [54]. In metaphor framing studies, participants are usually presented with a metaphorical (vs. non-metaphorical) description of a target issue and then asked to make a judgment or a decision on the target to check whether it is influenced by the metaphorical frame provided by the text. Thibodeau and Borodisky $[66, \mathrm{p} .1]$ stated that "even the subtlest instantiation of a metaphor (via a single word) can have a powerful influence over how people attempt to solve social problems", even when presented with possible alternatives after reading the text [67]. But later on, Steen and colleagues [64] addressed some criticisms to their studies, which actually presented to participants extended metaphors or anyway metaphors reinforced by possible metaphorical readings of other words in the text without a control (literal or "neutral framed") condition. Steen and colleagues therefore proposed a series of follow-up studies, also presenting texts with no additional support for the metaphoric frame, adding a non-metaphorical control condition, and previously measuring the political preferences of the participants. They found neither effects of the metaphorical frame nor of the metaphorical support on reasoning. They concluded that an increased media attention and/or a simple text exposure to the issue as a relevant social problem finally influenced the policy preferences. Interestingly, they also found that the (conventional) metaphors like "beast" and "virus" did not "surpass a nonmetaphorical frame in terms of prominence or attention" [64, p. 21], while the metaphorical support increases the activation, and thus the prominence of the metaphorical frame. They finally suggested that novelty, artfulness or deliberateness of a metaphor might play a major role in enhancing the metaphorical processing as well as the communicative effects of the text.

While Thibodeau and Borodisky [66] focused on conventional metaphors, Scherer and colleagues (2015) also presented texts with novel metaphors for their study on the metaphorical framing effect of flu description on vaccination intentions. They anyway found no significant difference on the metaphorical framing on vaccination intentions, "with novel and conventional metaphors all increasing vaccination intentions" [54, p. 44]. Though the metaphors were 
extended along the text via a relevant metaphorical property, the texts presented to participants were focused on the description of the flu in a reasoning situation that was consistent with the metaphorical frame. In other words, participants' disposition to get vaccinated was assessed via the reading of a text that did not bring participants to question the metaphorical frame and/or the relevant metaphorical property. As the results of the study showed, such reasoning scenarios do not change the (either against or in favor of vaccination) beliefs on vaccination participants already strongly held, while having some impact on people who occasionally got vaccination. However, in every-day life specific reasoning situations are widespread where laypeople question the (either metaphorical or literal) description of vaccination, possibly leading to undesired conclusions on vaccination as a collaborative endeavor. Some specific reasoning scenarios may indeed defeat conclusions about the necessity for vaccination to be collective, presenting further premises ranging from the phenomenon of single "free riders" to entire antivaccination communities. Such specific defeasible reasoning situations where vaccination is metaphorically described as collaborative have not been tested and are indeed relevant to understand whether and how metaphor interpretation interacts with the argumentative scaffolding of pro-vaccination texts. Especially when intentionally used, metaphors are supposed to be highly relevant in a variety of argumentative discourse structures [73; 74], but they have not yet been tested for their communicative power in defeasible reasoning situations, as required by a full understanding of vaccination as a collaborative phenomenon.

Reasoning about vaccination can thus be fruitfully approached by trying to highlight the role that certain premises play in it. In fact, depending on certain premises, a pattern of inference can be more or less exposed to uncertainty: ordinary reasoning is usually defeasible, but within a certain set of premises, there are premises that are in principle more likely to put the conclusion into question, or even defeat it $[29,44,45]$. Certain sets of premises can indeed be more or less consistent, and when slight (or even serious, depending on the context) inconsistencies show up, the reasoning can be exposed to uncertainty and the conclusion can be (temporarily) retracted. These premises may become, in these situations, potentially invalidating conditions for the reasoning process, possibly leading to a retraction of the conclusion. For example, consider a generic set of premises, such as the fact $\mathrm{X}$; the fact $\mathrm{Y}$; and its conclusion: the foreseen outcome $\mathrm{O}$. This set of premises $(\mathrm{X}, \mathrm{Y})$ can be seen as the unproblematic (or "safe") case, where the expected outcome $(\mathrm{O})$ is likely to happen. If we add a new premise, call it "the fact Z", which can alter the consistency of the set of premises, the reasoning task would get complicated, even requiring tentative solutions. These complications in the reasoning task require some means to explore the role of certain premises. Metaphor here 
is a potentially useful reasoning device to interpret the new premises, to facilitate and implicitly check the consistency of the premises within the set, and eventually accomplish the reasoning task: either by defeating the former conclusion or by temporarily retracting it waiting for further clues. However, no empirical study on the effects of metaphor concerning reasoning about vaccination, has been conducted. The study presented in the paper aims to fill this gap, by investigating whether a metaphor intentionally used to provide the readers with a perspective on vaccination as a collaborative health phenomenon, might enhance the communicative effects of a text based on reasoning about collective vaccination.

\section{Theoretical background}

The first assumption is that ordinary reasoning, and therefore also reasoning about vaccination, is exposed to uncertainty (section 2.1). The second assumption is that metaphor is a reasoning device to better understand the target, i.e. vaccination as a collective health phenomenon [15] (section 2.2).

\subsection{Uncertainty and reasoning in vaccine communication}

Vaccine hesitancy and vaccine communication present a number of challenges to doctors' expertise. Some of these challenges comprise reasoning about vaccination as a collective endeavor. Certain reasoning tasks, concerning sets of premises whose consistency is not fully explicit, usually require a major effort to evaluate the conclusions. These cases can be called uncertain scenarios. This type of reasoning is usually framed in terms of defeasible reasoning, and these premises are usually defined as defeaters, as they defeat the conclusion of the argument $[29,44,45]$. In uncertain reasoning scenarios, some relevant premises have the potential logical role of defeaters, even if they do not actually defeat any conclusion on the necessity of vaccination as a collective effort. We therefore treat these cases as uncertain scenarios where defeats, if any, are yet to be established. A reasoning is uncertain when the presence or absence of a certain premise (or set of premises) is in general liable to alter, or even to put into question, its conclusion. This means that its conclusion does not necessarily follow from certain premises: if the premise $\mathrm{P}$ usually allows to conclude $\mathrm{C}$, a further premise $\mathrm{F}$ may defeat that reasoning; so, while the inference from $\mathrm{P}$ to $\mathrm{C}$ is good in relatively easy scenarios, the eventual presence of $\mathrm{F}$ may defeat that transition, and $\mathrm{C}$ does not follow anymore, or at least it requires more effort on the side of the reasoner for proper assessment. 
The fact that a premise may defeat a reasoning, or make it uncertain, can be a problem as there is no warrant that this fact is known in advance. An example is the following: "collective vaccination is effective in assuring that an unvaccinated child stays safe; Dave is an unvaccinated child; hence, Dave can stay safe". This is a relatively certain scenario, and a quite easy-going reasoning task. However, if we add certain premises, this conclusion may become uncertain, and can even be temporarily retracted. For example, we can add these premises: "Dave lives in a community with a high rate of vaccine hesitancy; an unvaccinated person cannot stay safe in a context of vaccine hesitancy". These additional premises reshape the reasoning, to the point of defeating its conclusion. This reasoning would now involve a different series of steps: "collective vaccination is effective in assuring that an unvaccinated child stays safe; Dave is an unvaccinated child; Dave lives in a community with a high rate of vaccine hesitancy; an unvaccinated person cannot stay safe in a context of vaccine hesitancy"; hence, the conclusion "Dave can stay safe" does not follow anymore. The additional premises, that Dave lives in a community with a high rate of vaccine hesitancy and so forth, alter the set of premises, and defeat the conclusion that we formerly were entitled to draw [44, 45]. As this example shows, certain premises can systematically alter a more easier reasoning scenario making it uncertain and liable to be defeated. These potential complications can indeed lead to temporary retraction of a conclusion, or to its global defeat.

We can distinguish two main roles that premises may play in uncertain scenarios: (a) premises that are liable to undercut the inference to a conclusion $(\mathrm{U})$, as they may provide reasons that question the goodness of a conditional, and (b) premises that are liable to rebut the conclusion (R), as they may provide reasons that directly question a conclusion:

(a) The first role concerns the possibility of questioning the goodness of a general relation between certain premises and a particular conclusion. Consider a general connection between a premise and a conclusion: usually collective vaccination lets unvaccinated children stay safe. This can be rephrased as a conditional claim: if an unvaccinated child is protected by collective vaccination, then she can stay safe. We might question the goodness of this conditional: in principle everybody should be vaccinated and an unvaccinated child can stay safe as long as vaccination is collective. However, if everyone thinks that their child can be unvaccinated and anyway stay safe, the goodness of the inference will be undercut (U): vaccination would be no more collective and unvaccinated children could not stay safe. Paradoxically, as in the case of single "free riders", they can take advantage of vaccination without being vaccinated, as long as they do not undermine the need for vaccination to be collective. 
(b) The second role consists of a prima facie reason to directly question the conclusion of an inference. For example, Alice believes that collective vaccination protects also unvaccinated people and concludes that unvaccinated children can stay safe. However, Alice learns about Aldo, a child suffering from a serious pathology, who cannot get vaccinated and cannot stay safe since people around him are not vaccinated. Hence, the actual presence of unvaccinated people in Aldo's community is a fact that counts as a premise to be handled with care in reasoning about vaccination. If unvaccinated people are the majority, the premise can become the basis to directly rebut (R) Alice's conclusion.

The different roles of premises in reasoning about vaccination also shows that, depending on the proportion of unvaccinated people, our reasoning path can be more or less nuanced. Reasoning on a single unvaccinated child and reasoning on the fact that Aldo, the unvaccinated little boy, is surrounded by antivaccinists, makes a relevant difference. In the latter case, we can seriously reconsider the connection between collective vaccination and safety for unvaccinated individuals as reliable. So, premises that threaten to defeat a conditional are potentially more revisionary in scope, while premises liable to rebut a conclusion may highlight some local problems in our reasoning as we may discover that such conclusion actually does not follow [29].

Medical expertise involves navigating across a web of potential invalidating conditions, which create epistemically uncertain scenarios. This equals almost to a proviso that premises are to be handled with special care within these reasoning tasks. Therefore, medical expertise, in the context of uncertain reasoning tasks, consists of the abilities to identify the correct connections between safer and more uncertain premises, and to rule out the potential invalidating conditions for the expert's hypotheses (and not only in cases concerning vaccination). Many factors can be helpful in such uncertain scenarios: the usual route is provided by procedures of belief-revision between interlocutors, where their dialogue is an implicit means in order to check the mutual goodness and consistency of one's beliefs. This is a reliable way to understand how we manage to improve our reasoning skills, but it is not easy, and it is also fallible. However, an interesting alternative option may come from recent studies on metaphor as a reasoning device in argumentative texts.

\subsection{Metaphor as a reasoning device}

In the case of institutional communication aiming at improving the vaccination rates, it is of outstanding importance to let people understand how experts handle reasoning about 
vaccination in uncertain scenarios. Metaphor might be a good candidate to let people grasp how vaccination works at a collective level and reason about the many ways the individual adherence to the therapy might affect the collective endeavor of vaccination, in the perspective of a shared health responsibility. In this perspective, metaphors need to be carefully selected based on their potentiality to fit (or even enhance) the reasoning required by an argumentative discourse on diagnosis and consequent need for therapy adherence in the case of vaccine-preventable diseases. Based on traditional approaches to metaphor in science $[6 ; 26]$, we consider metaphor as a reasoning device guiding the readers along a path of inferences to a conclusion, which attributes to the target some relevant properties of the source $[41 ; 16]$.

Previous literature pointed out that selecting a source instead of another to construct a metaphor is never neutral in terms of the reasoning process it prompts (see [69] for a review), as it implicitly provides a specific perspective under which to interpret the target, "often including specific attitudes and evaluations" [57]. Most studies on metaphor framing are based on the conceptual theory of metaphor (CMT), whose authors claimed that metaphor is grounded in our embodied experience of the world [30] and therefore shapes how we reason about the world [31]. Far from being a mere embellishment of the text, metaphor proved to covertly influence how people reason and "gather information to make 'well-informed' decisions" [66, p. 10] about important and complex social issues, such as crime. Also, in the field of health communication, Hauser and Schwarz [25] found that, when compared to neutral frames, framing cancer as an enemy reduces patients' intentions to limit behaviors considered correlated to cancer likelihood. Interestingly, in the case of vaccination, Scherer and colleagues [54] found that other metaphorical frames of the flu (described as beast, riot, army or weed) did increase the intentions to get vaccinated when compared to a non-metaphorical frame (virus).

However, as previously pointed out $[22 ; 36 ; 39 ; 46]$, evidence was rarely found for knowledge transfer across domains unless a reasoner has some instruction to do so. On the contrary, within the domain of everyday dilemmas, strong framing effects were reported for the kind of reasoning required by the text (see for instance [72], on a dilemma involving a disease outbreak). Interestingly, in a metaphor-based task for the resolution of dilemmas, metaphors proved to have no (or even undesired) communicative effects, especially when increasing ambiguity or uncertain reasoning [50]. The specific line of reasoning required to the participants to solve the dilemma dominated over possible metaphorical inferences on the target: as the authors concluded, "the metaphor is unnecessary in cases in which a metaphor interferes with the reasoner's understanding process" [50, p. 61]. Under this respect, far from scaffolding and covertly driving reasoning as claimed by Thibodeau and colleagues [68], the reasoner's 
understanding process would rather drive the eventual effects of the metaphorical framing. Still, it is an open question whether and how the reasoning process and the metaphorical framing might creatively interact. For instance, in simple deductive tasks, Ervas and colleagues [17] showed that conventional metaphors, whose meaning is lexicalized and so familiar that goes unnoticed by participants, can be revitalized to hold a plausible conclusion of the argument, while novel metaphors, whose meaning is so new and creative that are fast recognized as metaphorical, rather lead to think about alternative conclusions.

Previous studies tested reasoning about "safe" scenarios where participants were asked to draw a conclusion, and checked where their inferences were influenced by the metaphorical description of the issue. Scherer and colleagues [54] tested the effects of the metaphorical framing of flu description on vaccine intentions, without considering different uncertain reasoning scenarios. No study on the effects of metaphors for vaccination on reasoning in uncertain scenarios has been conducted, even though it should be required in vaccine communication especially in times of vaccine hesitancy. Also, the results of corpus studies on metaphors' effects in other medical fields are mixed, questioning the role of metaphors as useful reasoning devices in health communication. Previous studies also looked at metaphor as a source of ambiguity and misunderstandings, highlighting missed alignments between experts' and patients' understanding of metaphors in consultations [58; 34]. Therefore, the question of the superiority of metaphor over literal language in health communication remains open. It is still unclear when and how metaphors might be effective reasoning devices to improve patients' understanding of a specific diagnostic process, as for instance in the case of vaccination, as well as adherence to therapy.

\section{The Study}

A promising (but still controversial, see $[12 ; 20 ; 21 ; 37 ; 11]$ and $[2]$ for criticism) venue for research on the effects of metaphor in health communication might come from the deliberate metaphor theory (DMT) $[62,63,65]$, whose main proponent revisited CMT, arguing that what counts in communication is not embodied metaphors per se, but the deliberate use of a metaphor to draw attention on a target issue with specific communicative aims, i.e. the intentional use of a metaphor as a metaphor in (argumentative) discourse. For instance, a novel deliberate metaphor (especially when direct, as for instance when introduced by "like" explicitly 
prompting a comparison between domains) can be fruitfully employed as a rebuttal analogy in an argumentative discourse, while a conventional metaphor would not serve this job [73].

As proposed in a previous study [52], a metaphor might be intentionally selected for its properties as long as it fits the argumentative aims of health communication, to better explain a (collective) health problem, to make some reasons for a diagnosis clearer, to offer an alternative view on an illness (or on a system of commonplaces associated with the illness) and/or to solicit for a belief revision. In this perspective, an argumentatively apt metaphor, i.e. one whose properties favor the reasoning process required to understand the health issue, might reveal to be crucial to both diagnosis understanding and adherence to therapy. As the effectiveness of health communication is intrinsically linked with patient trust [18; 71], an argumentatively apt metaphor use might turn out to be important not only for a successful expert-patient relationship but also to the trust in experts and the institution they represent. However, to the best of our knowledge, no empirical study on the effects of metaphor use on trust on experts and institutions in the case of vaccine communication in uncertain scenarios has been conducted.

\subsection{Experimental Hypotheses}

We conducted a study to investigate whether and when a novel metaphor ("the beehive", see $5 ; 15)$, extended via the relevant property ("cooperative") for argumentative discourse about vaccination in uncertain scenarios, can be an effective reasoning and communicative device for laypeople's understanding of vaccination as a vital collective endeavor. The study aims at understanding the communicative effects of metaphors in uncertain reasoning situations, in terms of persuasion, emotional impact, trust in experts/institutions, and vaccination intentions. We proposed the following main hypotheses:

H1: A novel metaphor for vaccination, extended via a relevant property for the reasoning task, entails stronger communicative effects than its literal counterpart, as it is explicitly intended to make collective vaccination dynamic more intelligible for laypeople.

$\mathrm{H} 2$ : Reasoning situations concerning uncertain scenarios enhance the comprehension of the text on vaccination when compared to safe reasoning scenarios, as they make possible defeating conditions (not envisaged in safe scenarios) explicit. 
We expected that participants grasped the importance of collective vaccination and healthrelated behaviors, especially in uncertain scenarios with potential rebutting defeaters, which offer a prima facie reason (a fact) to directly question the conclusion.

As extended metaphors were explicitly intended to highlight the target (vaccination), under an alternative perspective, they drive the reader's attention toward the conclusion, especially in the case of premises that are more liable to make the reasoning scenario uncertain. This function should not be achieved by their literal counterparts, as they do not help per se defeasible reasoning in providing an alternative perspective. We therefore hypothesized that the metaphorical frame interacts with the reasoning process type, in the following way:

H3: Extended metaphors are more effective as both communicative and reasoning devices than their literal counterparts in uncertain scenarios, where reasoning is usually defeasible. Especially in the case of possible undercutting defeaters, concerning a potential withdrawal of the main inference, the explicit relevant property of the metaphor for defeasible reasoning might make it easier for the interlocutor to discern whether the inference is good or bad. Thus, when the metaphor is extended via a relevant property to support or even enhance the defeasible reasoning process on vaccination, it can better achieve some communicative effects in the argumentative discourse, in terms of comprehension, persuasion, and trust in the experts (and the institutions they represent).

\subsection{Design}

The empirical study had a $3 \times 2$ experimental design: 3 "reasoning" conditions (a. safe reasoning scenarios; b. uncertain reasoning scenarios - undercutting-type, $U$; c. uncertain reasoning scenarios - rebutting-type, $\mathrm{R}) \times 2$ "metaphorical frame" conditions (a. metaphor; $b$. literal counterpart).

Six group of participants were therefore provided with an argumentative text on vaccination in the following six conditions:

1) texts presenting a safe reasoning scenario with either a metaphor (MS) or its literal counterpart (LS);

2) texts presenting an uncertain reasoning scenario (undercutting-type) with either a metaphor (MU) or its literal counterpart (LU);

3) texts presenting an uncertain reasoning scenario (rebutting-type) with either a metaphor (MR) or its literal counterpart (LR). 


\subsection{Participants}

A total of 196 adults $\left(152 \mathrm{~F}, 44 \mathrm{M} ; \mathrm{M}_{\mathrm{age}}=27.97\right.$ years; $\left.\mathrm{SD}_{\mathrm{age}}=10.40\right)$ participated in the study. All participants had Italian as their first language and signed an informed consent form indicating that they understood the nature of their participation in the study, which was approved by the Ethics Committee of the University of Cagliari (n. 0107679, 05/06/2020). A description of the six groups of participants, each assigned with a single condition, is provided in Table 1.

Table 1. Participants' demographic table

\begin{tabular}{r|cccccc} 
Condition & $\boldsymbol{L S}$ & $\boldsymbol{L U}$ & $\boldsymbol{L R}$ & $\boldsymbol{M S}$ & $\boldsymbol{M U}$ & $\boldsymbol{M R}$ \\
\hline Total Number & 32 & 31 & 33 & 30 & 33 & 37 \\
Gender & $24 \mathrm{~F} / 8 \mathrm{M}$ & $24 \mathrm{~F} / 7 \mathrm{M}$ & $24 \mathrm{~F} / 9 \mathrm{M}$ & $22 \mathrm{~F} / 8 \mathrm{M}$ & $27 \mathrm{~F} / 6 \mathrm{M}$ & $31 \mathrm{~F} / 6 \mathrm{M}$ \\
Mean(age) & 32.25 & 31.64 & 27.88 & 26.70 & 22.97 & 26.76 \\
Standard Deviation(age) & 10.03 & 10.32 & 10.14 & 10.92 & 5.72 & 11.86 \\
Vaccinated in the last 3 years & $25 \%$ & $19,4 \%$ & $36,4 \%$ & $40 \%$ & $39,4 \%$ & $40,5 \%$ \\
Vaccinated for seasonal flu in the last 3 years & $12,5 \%$ & $19,4 \%$ & $9,1 \%$ & $13,3 \%$ & $15,2 \%$ & $10,8 \%$
\end{tabular}

Most participants declared to gather information about vaccination consulting a medical competent expert $(43,1 \%)$ or via institutional websites $(31,8 \%)$; a minority via non-institutional websites and social networks $(3,7 \%)$, informal discourses with relatives or friends $(5,5 \%)$, scientific journals $(6,2 \%)$. Some participants declared to be not informed at all about vaccination $(6,9 \%)$.

\subsection{Material}

Previous literature showed that metaphors would encourage people to draw inferences consistent with the frame provided by the text, especially when the metaphor is put at the beginning, thus activating the source domain knowledge from where to draw the inferences [68], and extended along the text [28; 70]. We therefore presented to participants texts where the metaphor occurs as soon as the description of vaccination is introduced, and is extended via its relevant property ("collective" in the literal conditions vs. "collaborative" in the metaphorical conditions) for reasoning about potentially defeating scenarios. The stimulus material consisted of six argumentative texts on vaccination (one per condition, see Supplementary material, Table 1 for the material in Italian). Table 2 presents the texts translated into English, with the potential defeaters in italics and the extended metaphor in bold (as well as its literal counterpart in the literal conditions). 
Table 2. Argumentative texts on vaccination (translation into English)

\begin{tabular}{|c|c|c|c|}
\hline \multicolumn{2}{|r|}{ Safe reasoning $(S)$} & $\begin{array}{l}\text { Uncertain reasoning - } \\
\text { Undercutting-type (U) }\end{array}$ & $\begin{array}{l}\text { Uncertain reasoning - } \\
\text { Rebutting-type }(R)\end{array}$ \\
\hline $\begin{array}{r}\text { Literal } \\
(L)\end{array}$ & $\begin{array}{l}\text { The expert talked about collective } \\
\text { immunity concerning viral epidemics } \\
\text { and told that the vaccination of } \\
\text { everyone is a fundamental } \\
\text { requirement against contagion. In } \\
\text { particular, the doctor insisted on } \\
\text { defining the collective effort for } \\
\text { everyone's vaccination as that of the } \\
\text { components of a group, where the } \\
\text { collective counts more than the } \\
\text { individual. The speech concerned the } \\
\text { idea of collective commitment of all } \\
\text { citizens. The expert concluded } \\
\text { emphasizing how much we are } \\
\text { dependent on each other in the } \\
\text { context of sanitary emergencies. }\end{array}$ & $\begin{array}{l}\text { The expert talked about collective } \\
\text { immunity concerning viral epidemics } \\
\text { and told that the vaccination of } \\
\text { everyone is a fundamental requirement } \\
\text { against contagion. In particular, the } \\
\text { doctor insisted on defining the } \\
\text { collective effort for everyone's } \\
\text { vaccination as that of the components } \\
\text { of a group, where the collective counts } \\
\text { more than the individual. The speech } \\
\text { concerned the idea of collective } \\
\text { commitment of all citizens. The expert } \\
\text { stressed that even an unvaccinated child } \\
\text { can stay safe if people around him are } \\
\text { vaccinated. The expert concluded } \\
\text { emphasizing how much we are } \\
\text { dependent on each other in the context } \\
\text { of sanitary emergencies. }\end{array}$ & $\begin{array}{l}\text { The expert talked about collective } \\
\text { immunity concerning viral epidemics } \\
\text { and told that the vaccination of } \\
\text { everyone is a fundamental requirement } \\
\text { against contagion. In particular, the } \\
\text { doctor insisted on defining the collective } \\
\text { effort for everyone's vaccination as that } \\
\text { of the components of a group, where } \\
\text { the collective counts more than the } \\
\text { individual. The speech concerned the } \\
\text { idea of collective commitment of all } \\
\text { citizens. The expert provided the example } \\
\text { of Aldo, a child suffering from a serious } \\
\text { pathology, who cannot get vaccinated } \\
\text { and cannot stay safe since people around } \\
\text { him are not vaccinated. The expert } \\
\text { concluded emphasizing how much we } \\
\text { are dependent on each other in the } \\
\text { context of sanitary emergencies. }\end{array}$ \\
\hline $\begin{array}{r}\text { Metaphorical } \\
(M)\end{array}$ & $\begin{array}{l}\text { The expert talked about collective } \\
\text { immunity concerning viral epidemics } \\
\text { and told that the vaccination of } \\
\text { everyone is a fundamental } \\
\text { requirement against contagion. In } \\
\text { particular, the doctor insisted on } \\
\text { defining the collective effort for } \\
\text { everyone's vaccination as that of the } \\
\text { bees in a beehive, where the } \\
\text { collective counts more than the } \\
\text { individual. The speech concerned the } \\
\text { idea of collaborative commitment of } \\
\text { all citizens. The expert concluded } \\
\text { emphasizing how much we are } \\
\text { dependent on each other in the } \\
\text { context of health emergencies. }\end{array}$ & $\begin{array}{l}\text { The expert talked about collective } \\
\text { immunity concerning viral epidemics } \\
\text { and told that the vaccination of } \\
\text { everyone is a fundamental requirement } \\
\text { against contagion. In particular, the } \\
\text { doctor insisted on defining the } \\
\text { collective effort for everyone's } \\
\text { vaccination as that of the bees in a } \\
\text { beehive, where the collective counts } \\
\text { more than the individual. The speech } \\
\text { concerned the idea of collaborative } \\
\text { commitment of all citizens. The expert } \\
\text { stressed that even an unvaccinated child } \\
\text { can stay safe if people around him are } \\
\text { vaccinated. The expert concluded } \\
\text { emphasizing how much we are } \\
\text { dependent on each other in the context } \\
\text { of health emergencies. }\end{array}$ & $\begin{array}{l}\text { The expert talked about collective } \\
\text { immunity concerning viral epidemics } \\
\text { and told that the vaccination of } \\
\text { everyone is a fundamental requirement } \\
\text { against contagion. In particular, the } \\
\text { doctor insisted on defining the collective } \\
\text { effort for everyone's vaccination as that } \\
\text { of the bees in a beehive, where the } \\
\text { collective counts more than the } \\
\text { individual. The speech concerned the } \\
\text { idea of collaborative commitment of all } \\
\text { citizens. The expert provided the example } \\
\text { of Aldo, a child suffering from a serious } \\
\text { pathology, who cannot get vaccinated } \\
\text { and cannot stay safe since people around } \\
\text { him are not vaccinated. The expert } \\
\text { concluded emphasizing how much we } \\
\text { are dependent on each other in the } \\
\text { context of health emergencies. }\end{array}$ \\
\hline
\end{tabular}

\subsection{Procedure}

We collected the data through six different online forms, one for each condition. After the participants signed the informed consent, the form gathered information about gender, age, language, and education. The participants were then asked to read the instructions and fill out a questionnaire, with answers on a 1 to 7 Likert Scale, ranging from 1 as "not at all" to 7 as "very much".

In the first part of the questionnaire, all the forms presented the conclusion of the argumentative texts as a stand-alone sentence (the same for all conditions): "In the context of health emergencies we are dependent on each other". The participants were asked to rate how much they agree with the statement. Then, on a separate screen, the questionnaire presented the same sentence as the conclusion of the argumentative text (different for each condition, see Table 2), asking the participants how much it was logically acceptable, when considering the premises of the text. 
In the second part of the questionnaire, all the forms presented the relative argumentative text followed by a series of questions, each one on a separate screen. The questions were focused on text comprehension as well as on its perceived communicative effects (see Table 3 for Measures) at individual and collective level, as the evaluation of the argumentative texts on vaccination could involve reasoning not only on possible personal vaccination behavior but also its link with the possible collective vaccination behavior.

Table 3. Description of measures and related response categories

\begin{tabular}{|c|c|c|}
\hline Measures & Response categories & Description \\
\hline \multirow[t]{2}{*}{ Reasoning } & Agreement & $\begin{array}{l}\text { Participants indicated their level of agreement with the statement (= the } \\
\text { conclusion as a single sentence). }\end{array}$ \\
\hline & Logical acceptability & $\begin{array}{l}\text { Participants indicated whether the conclusion was logically acceptable, given } \\
\text { the premises of the text. }\end{array}$ \\
\hline \multirow[t]{2}{*}{ Comprehension } & Understandability & $\begin{array}{l}\text { Participants indicated how much the text was easy to understand (at the } \\
\text { individual and collective level). }\end{array}$ \\
\hline & Ambiguity & Participants indicated whether they found meaning ambiguities in the text. \\
\hline \multirow[t]{3}{*}{ Persuasion } & Emotional impact & $\begin{array}{l}\text { Participants indicated how much they found the advice presented in the text } \\
\text { emotionally appealing (at the individual and collective level). }\end{array}$ \\
\hline & Convincingness & $\begin{array}{l}\text { Participants indicated how much they found convincing (at the individual and } \\
\text { collective level) the advice presented in the text. }\end{array}$ \\
\hline & Safety & $\begin{array}{l}\text { Participants indicated the feeling of safety (at the individual and collective } \\
\text { level) in a health situation like that described in the text. }\end{array}$ \\
\hline \multirow[t]{2}{*}{$\begin{array}{r}\text { Real-life } \\
\text { Experience }\end{array}$} & Control & $\begin{array}{l}\text { Participants indicated the feeling of control (at the individual and the collective } \\
\text { level) in the health situation described in the text. }\end{array}$ \\
\hline & Commitment & $\begin{array}{l}\text { Participants indicated the level of commitment to vaccination (at the individual } \\
\text { and collective level). }\end{array}$ \\
\hline \multirow[t]{3}{*}{ Trust } & Trust in Experts & $\begin{array}{l}\text { Participants indicated the level of trust in experts (at the individual and } \\
\text { collective level). }\end{array}$ \\
\hline & $\begin{array}{l}\text { Uptake of the } \\
\text { expert's' advice }\end{array}$ & $\begin{array}{l}\text { Participants indicated the (individual and collective) level of uptake of the } \\
\text { expert's advice }\end{array}$ \\
\hline & Trust in Institutions & Participants indicated the level of trust in institutions \\
\hline $\begin{array}{r}\text { Vaccination } \\
\text { Intentions }\end{array}$ & $\begin{array}{l}\text { Vaccination } \\
\text { Intentions }\end{array}$ & $\begin{array}{l}\text { Participants indicated the likelihood that they would get vaccinated in the } \\
\text { upcoming winter season. }\end{array}$ \\
\hline
\end{tabular}

First of all, we aimed to measure whether metaphor actually made the texts easier to understand, though introducing a meaning ambiguity (the literal meaning vs. the metaphorical meaning of the source). We then selected the measures, and related questions, from previous literature on the metaphorical framing effects on vaccination [54] in terms of persuasion, perceived control, and vaccination intentions. In light of possible effects of extended metaphor on source credibility and trust $[1,9,10,48]$, we added specific measures for participants' trust on the expert's advice, on experts and the institutions they represent, we deemed to be highly relevant for vaccine communication.

The final section of the questionnaire gathered information about participants' previous general vaccination behavior and beliefs about (flu) vaccination, the impact of COVID-19 and an eventual COVID-19 vaccination on their lives (see Table 2 in Supplementary Material for the questionnaire). 


\subsection{Results}

All the data are collected at the following OSF address. Mean and standard deviation for each condition and response category are presented in Table 4.

Table 4. Mean (M) and standard deviation (SD) for each condition and response category

\begin{tabular}{|c|c|c|c|c|c|c|c|c|c|c|c|c|}
\hline \multirow{4}{*}{ Condition } & \multicolumn{6}{|c|}{ Literal } & \multicolumn{6}{|c|}{ Metaphorical } \\
\hline & \multirow{2}{*}{\multicolumn{2}{|c|}{$\begin{array}{l}\text { Standard } \\
\text { reasoning }\end{array}$}} & \multicolumn{4}{|c|}{ Defeasible reasoning } & \multirow{2}{*}{\multicolumn{2}{|c|}{$\begin{array}{l}\text { Standard } \\
\text { reasoning }\end{array}$}} & \multicolumn{4}{|c|}{ Defeasible reasoning } \\
\hline & & & \multirow{2}{*}{\multicolumn{2}{|c|}{$\begin{array}{c}\text { Undercutting } \\
\text { LU }\end{array}$}} & \multirow{2}{*}{\multicolumn{2}{|c|}{$\begin{array}{c}\text { Rebutting } \\
\text { LR } \\
\end{array}$}} & & & \multirow{2}{*}{\multicolumn{2}{|c|}{$\frac{\text { Undercutting }}{\text { MU }}$}} & \multirow{2}{*}{\multicolumn{2}{|c|}{$\begin{array}{c}\text { Rebutting } \\
\text { MR }\end{array}$}} \\
\hline & \multicolumn{2}{|c|}{ LS } & & & & & \multicolumn{2}{|c|}{ MS } & & & & \\
\hline Response category & $\mathbf{M}$ & SD & $\mathbf{M}$ & SD & $\mathbf{M}$ & SD & $\mathbf{M}$ & SD & \multicolumn{2}{|c|}{$\begin{array}{ll}M & \text { SD }\end{array}$} & $\mathbf{M}$ & SD \\
\hline Agreement & 5,63 & 1,62 & 6,26 & 1,21 & 6,12 & 0,93 & 5,87 & 1,50 & 5,97 & 1,02 & 6,16 & 1,17 \\
\hline Logical Acceptability & 5,44 & 1,68 & 5,61 & 1,63 & 6,24 & 0,97 & 6,07 & 1,53 & 6,00 & 1,37 & 6,30 & 1,29 \\
\hline Understandability_I & 5,69 & 1,64 & 6,45 & 0,81 & 6,21 & 1,08 & 6,37 & 1,25 & 6,64 & 0,78 & 6,70 & 0,74 \\
\hline Understandability_C & 4,00 & 1,55 & 4,35 & 1,28 & 4,55 & 1,28 & 4,57 & 1,33 & 5,15 & 1,15 & 5,14 & 1,27 \\
\hline Ambiguity & 3,34 & 1,86 & 2,55 & 1,71 & 2,00 & 1,64 & 2,73 & 2,29 & 2,48 & 1,80 & 1,73 & 1,15 \\
\hline Emotional impact_I & 3,53 & 2,27 & 3,58 & 1,88 & 4,12 & 2,00 & 3,40 & 1,99 & 4,27 & 1,68 & 4,86 & 1,72 \\
\hline Emotional impact_C & 4,00 & 1,80 & 3,74 & 1,73 & 4,48 & 1,48 & 3,97 & 1,22 & 4,42 & 1,32 & 5,19 & 1,31 \\
\hline Convincingness_I & 4,03 & 1,77 & 4,19 & 1,82 & 5,15 & 1,37 & 5,00 & 1,39 & 5,52 & 1,33 & 5,35 & 1,48 \\
\hline Convincingness_C & 3,97 & 1,51 & 4,42 & 1,52 & 4,79 & 1,32 & 4,47 & 1,07 & 5,03 & 1,13 & 5,24 & 0,95 \\
\hline Safety_I & 3,56 & 1,76 & 3,45 & 1,96 & 4,03 & 1,38 & 4,30 & 1,62 & 5,12 & 1,73 & 4,16 & 1,91 \\
\hline Safety_C & 3,56 & 1,27 & 4,00 & 1,69 & 3,97 & 1,24 & 3,83 & 1,42 & 4,76 & 1,35 & 4,27 & 1,17 \\
\hline Control_I & 4,25 & 2,14 & 4,16 & 2,13 & 4,09 & 1,59 & 4,50 & 1,72 & 5,03 & 1,51 & 4,54 & 1,54 \\
\hline Control_C & 3,88 & 1,66 & 4,87 & 1,48 & 4,82 & 1,47 & 5,00 & 1,72 & 4,85 & 1,52 & 5,19 & 1,52 \\
\hline Commitment_I & 5,38 & 1,79 & 5,97 & 1,80 & 6,42 & 1,09 & 5,77 & 1,92 & 6,45 & 1,23 & 5,73 & 2,02 \\
\hline Commitment_C & 5,88 & 1,84 & 6,26 & 1,59 & 6,79 & 0,48 & 6,10 & 1,54 & 6,61 & 0,79 & 6,54 & 0,93 \\
\hline Trust in Experts_I & 4,69 & 1,87 & 5,00 & 2,39 & 5,82 & 1,07 & 5,60 & 1,73 & 5,73 & 1,26 & 5,30 & 1,79 \\
\hline Trust in Experts_C & 4,28 & 1,33 & 4,23 & 1,48 & 4,52 & 1,03 & 4,47 & 0,94 & 5,06 & 0,93 & 4,84 & 0,83 \\
\hline Uptake of Experts' Adv._I & 4,91 & 2,02 & 5,23 & 2,17 & 6,21 & 0,93 & 5,83 & 1,62 & 6,15 & 1,25 & 5,65 & 1,92 \\
\hline Uptake of Experts' Adv_C & 3,81 & 1,38 & 4,06 & 1,41 & 4,36 & 1,03 & 4,07 & 0,98 & 4,88 & 1,02 & 4,30 & 0,70 \\
\hline Trust in Institutions_I & 5,13 & 1,60 & 5,13 & 2,17 & 5,70 & 0,98 & 5,33 & 1,40 & 5,76 & 1,35 & 5,16 & 1,72 \\
\hline Trust in Institutions_C & 4,00 & 1,41 & 3,77 & 1,61 & 3,79 & 1,11 & 3,60 & 1,25 & 4,55 & 1,15 & 3,76 & 1,14 \\
\hline Vaccination Intentions_I & 3,75 & 2,48 & 4,45 & 2,34 & 4,82 & 1,84 & 4,50 & 2,26 & 5,09 & 1,70 & 5,08 & 2,02 \\
\hline Vaccination Intentions_C & 4,34 & 1,94 & 4,94 & 2,00 & 5,30 & 1,40 & 4,83 & 1,90 & 5,36 & 1,54 & 5,30 & 1,87 \\
\hline
\end{tabular}

A multivariate analysis was performed on the data, having the metaphorical and reasoning structure as factors and the response categories in Table 3 as dependent variables, to assess the effects of the metaphorical frame and the reasoning situation, as well as the possible interaction effect of the two factors. The analysis reveals an overall main effect of both the metaphorical frame $\left(\mathrm{F}_{(23,168)}=2.10 ; p=0.004\right)$ and the reasoning scenario $\left(\mathrm{F}_{(46,336)}=2.01 ; p<0.001\right)$, but no interaction effect $\left(\mathrm{F}_{(46,336)}=1.13 ; p=0.275\right)$. The main effect of the metaphorical frame is due to the fact that participants' generally attributed higher score for all response categories (except for "Ambiguity") when vaccination was described metaphorically rather than literally. The main effect of the reasoning condition is due to the fact that participants' generally attributed higher scores for all response categories (except for "Ambiguity"), when the reasoning scenario was uncertain rather than safe. The results of the multivariate analysis did not significantly change when having age, education, personal opinion on vaccination, beliefs on the power of great pharmaceutical companies or overall Covid-19 impact as covariates. 
Furthermore, the univariate analysis for each response category showed that the metaphorical framing effect was significant especially for the overall text understandability (both at an individual, $p=0.003$, and at a collective level, $p<0.001$ ), the emotional impact (even though only at a collective level, $p=0.023$ ), the convincingness of the text (both at an individual, $p<0.001$, and at a collective level, $p=0.003$ ), the perceived safety (both at an individual, $p<0.001$, and at a collective level, $p=0.020$ ), the feeling of control over the health situation (both at an individual, $p=0.041$, and at a collective level, $p=0.027$ ), collective trust in expertise and uptake of experts' advice (respectively, $p=0.004$ and $p=0.034$ ). No significant effect of the metaphorical frame was found on participants' responses for the other categories (see Table 5). The main effect of the reasoning structure was instead found especially for participants' perceived ambiguity of meaning $(p<0.001)$, but also for the overall text understandability (both at an individual, $p=0.018$, and at a collective level, $p=0.038$ ), the convincingness of the text (both at an individual, $p=0.028$, and at a collective level, $p=0.002$ ), the collective perceived safety, commitment to vaccination and uptake of experts' advice (respectively, $p=0.019$, $p=0.011$ and $p=0.021$ ). No significant effect of the reasoning structure was found for the other response categories (see Table 5).

Table 5. Univariate analysis results for each response category

\begin{tabular}{|c|c|c|c|c|c|c|c|}
\hline & & \multicolumn{2}{|c|}{ Metaphorical frame } & \multicolumn{2}{|c|}{ Reasoning structure } & \multicolumn{2}{|c|}{$\begin{array}{c}\text { Metaphorical frame } * \\
\text { Reasoning structure }\end{array}$} \\
\hline \multicolumn{2}{|c|}{ Response category } & F value & $p$ value & F value & $p$ value & F value & $p$ value \\
\hline \multirow{3}{*}{ Reasoning } & Agreement & 0.00310 & 0.956 & 1.99659 & 0.139 & 0.71540 & 0.490 \\
\hline & Logical Acceptability & 3.11909 & 0.079 & 2.62490 & 0.075 & 0.67800 & 0.509 \\
\hline & Understandability_I & 8.98316 & 0.003 & 4.12036 & 0.018 & 0.83252 & 0.437 \\
\hline \multirow[t]{4}{*}{ Comprehension } & ${ }_{\text {Understandability_C }}$ & 12.68773 & $<.001$ & 3.31637 & 0.038 & 0.14949 & 0.861 \\
\hline & Ambiguity & 1.89570 & 0.170 & 7.47275 & $<.001$ & 0.38954 & 0.678 \\
\hline & Emotional impact_I & 3.04088 & 0.083 & 4.70698 & 0.010 & 1.03368 & 0.358 \\
\hline & Emotional impact_C & 5.23688 & 0.023 & 6.62781 & 0.002 & 1.26382 & 0.285 \\
\hline \multirow{5}{*}{ Persuasion } & Convincingness_I & 14.39435 & $<.001$ & 3.63936 & 0.028 & 2.35369 & 0.098 \\
\hline & Convincingness_C & 9.08635 & 0.003 & 6.61137 & 0.002 & 0.06661 & 0.936 \\
\hline & Safety_I & 11.20971 & $<.001$ & 0.71320 & 0.491 & 3.28595 & 0.040 \\
\hline & Safety_C & 5.52902 & 0.020 & 4.03805 & 0.019 & 0.64919 & 0.524 \\
\hline & Control_I & 4.22971 & 0.041 & 0.47013 & 0.626 & 0.49844 & 0.608 \\
\hline \multirow{5}{*}{$\begin{array}{l}\text { Real life } \\
\text { experience }\end{array}$} & Control_C & 4.99941 & 0.027 & 2.33962 & 0.099 & 2.18849 & 0.115 \\
\hline & Commitment_I & 0.04849 & 0.826 & 2.57400 & 0.079 & 2.56612 & 0.079 \\
\hline & Commitment_C & 0.41618 & 0.520 & 4.64279 & 0.011 & 1.02437 & 0.361 \\
\hline & Trust in Experts_I & 2.02899 & 0.156 & 0.87154 & 0.420 & 3.39243 & 0.036 \\
\hline & Trust in Experts_C & 8.33194 & 0.004 & 1.39753 & 0.250 & 1.52526 & 0.220 \\
\hline \multirow{4}{*}{ Trust } & Uptake of Experts' Adv._I & 2.84422 & 0.093 & 1.66849 & 0.191 & 4.26775 & 0.015 \\
\hline & Uptake of Experts' $A d v_{-} C$ & 4.55648 & 0.034 & 3.94957 & 0.021 & 2.69833 & 0.070 \\
\hline & Trust in Institutions_I & 0.14898 & 0.700 & 0.36376 & 0.696 & 2.33794 & 0.099 \\
\hline & Trust in Institutions_C & 0.39876 & 0.528 & 1.93501 & 0.147 & 3.44751 & 0.034 \\
\hline \multirow{2}{*}{$\begin{array}{l}\text { Vaccination } \\
\text { Intentions }\end{array}$} & Vaccination Intentions_I & 3.51225 & 0.062 & 2.69444 & 0.070 & 0.24375 & 0.784 \\
\hline & Vaccination Intentions_C $C$ & 1.52883 & 0.218 & 2.81748 & 0.062 & 0.38386 & 0.682 \\
\hline
\end{tabular}


The ANOVA post hoc analysis revealed a significant difference between safe reasoning and uncertain reasoning scenarios with rebutting defeaters for both the perceived meaning ambiguity $(\mathrm{t}=3.83 ; p<0.001)$ and the individual/collective persuasion categories (convincingness: $\mathrm{t}=-2.73 ; p=0.019$ and $\mathrm{t}=-3.67 ; p<0.001$; emotional impact: $\mathrm{t}=-3.05 ; p=0.007$ and $\mathrm{t}=-3.28 ; p=0.003$, respectively at individual and collective level). Rebutting reasoning scenarios were indeed perceived as less ambiguous, more convincing, and emotionally appealing than the safe reasoning situations, at both a personal and collective level. Only at the collective level a significant difference was revealed between safe and uncertain reasoning conditions (with rebutting defeaters) for both understandability $(\mathrm{t}=-2.49 ; p=0.036)$ and commitment to vaccination $(\mathrm{t}=-3.03 ; p=0.008)$. For both response categories, participants assigned higher value responses in the case of uncertain reasoning conditions with rebutting defeaters when compared to safe reasoning conditions. On the contrary, a significant difference was revealed between safe reasoning and uncertain reasoning with undercutting defeaters for both the collective perceived safety $(\mathrm{t}=-2.74 ; p=0.018)$ and the collective uptake of experts' advice ( $\mathrm{t}=-2.74 ; p=0.018)$. For both response categories, participants assigned higher value responses in the case of uncertain reasoning scenarios with undercutting defeaters when compared to safe reasoning scenarios.

Neither a main metaphorical framing effect nor a main reasoning structure effect was found on participants' acceptance of the conclusion, personal and collective trust in institutions, and vaccination intentions (see table 4). The univariate analysis for each response category showed instead a significant interaction of the metaphorical frame with the reasoning structure of the text on the personal feeling of safety $(p=0.040)$, individual trust in expertise and uptake of experts' advice (respectively, $p=0.036$ and $p=0.015$ ), and collective trust in institution $(p=0.034)$. Post hoc analysis revealed that the interaction effect on the personal feeling of safety was due to the significant difference between LS and MU conditions ( $p=0.005)$, and LU and MU conditions $(p=0.002)$ (Fig 1). As to what concerns the uptake of experts' advice, the interaction effect was due to a significant difference between LS and MU conditions ( $p=0.005)$, and LS and LR conditions $(p=0.028)$. As to what concerns collective trust in institutions, the interaction effect was due to a significant difference between MS and MU conditions $(p=0.045)$ (Fig 2). 

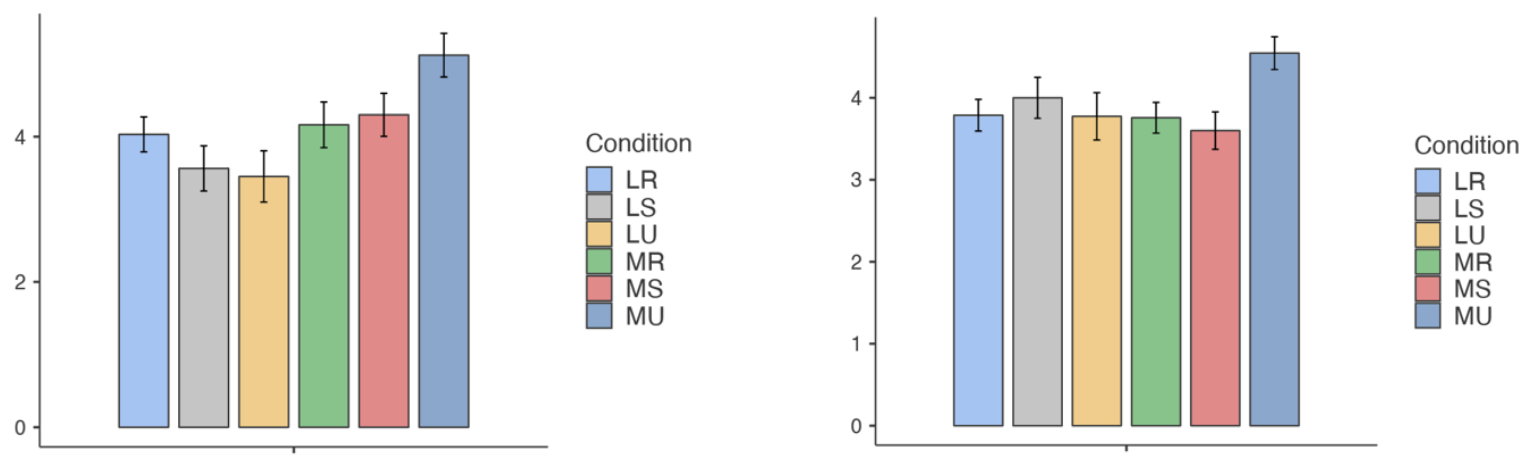

\section{Discussion}

Metaphor proves to be very useful to let laypeople understand a complex health issue in the case of vaccination: when compared to literal descriptions of vaccination, the beehive metaphor makes the message easier to understand. The results confirm previous literature as to what concerns the general communicative potential of metaphor [61; 42]: independently of the reasoning structure of the text, participants thought that the metaphorical description of vaccination could be more persuasive not only for themselves, but also for the majority of people, especially for what concerns the emotional impact. Metaphors can indeed have many functions in both interpersonal and mass communication and be mediated by multiple psychological mechanisms. First of all, metaphor activates information directly connected to the communication topic, as it attributes some relevant properties of the source to the target. In the process, it can favor the understanding of the communication topic, but also influence people's attitudes and emotive evaluation toward the communication topic [70; 14]. In the case of vaccine communication, participants' attitude and evaluations were indeed influenced by the metaphorical description of vaccination as a collaborative endeavor, especially for what concerned their perceived feeling of safety and control over the health situation at both a personal and collective level. The first main hypothesis (H1) that extended metaphors, explicitly intended to promote the vaccination uptake via a relevant property ("collaborative") for the argumentative text, elicit stronger communicative effects than its literal counterpart, is thereby confirmed.

The results also confirmed the second main hypothesis (H2) that uncertain reasoning scenarios are relevant for the comprehension of the text on vaccination. Not only the overall understandability of the text was higher in uncertain reasoning scenarios when compared to 
safe reasoning scenarios, but also its convincingness at both an individual and collective level. Texts based on an uncertain reasoning structure make explicit possible defeaters which are instead not envisaged in safe scenarios. Making possible defeating conditions explicit, texts based on uncertain reasoning structure allow a better grasp of how vaccination works, resulting even clearer than texts based on a safe reasoning structure. Besides anticipating possible defeaters, the texts based on uncertain reasoning scenarios propose a solution in the conclusion at the collective level, thus enhancing also the collective feeling of perceived safety, and the possible collective commitment and adherence to vaccination.

The persuasive effect of uncertain reasoning scenarios is significantly stronger than the safe one in the case of rebutting defeaters. Texts based on uncertain scenarios via rebutting defeaters are perceived not only as more understandable and leading to vaccination commitment at a personal level, but also as more convincing and emotionally appealing than the safe reasoning situations, at both a personal and collective level. These texts were indeed based on the single story of the child, Aldo, "suffering from a serious pathology, who cannot get vaccinated and cannot stay safe since people around him are not vaccinated". Drawing on a concrete and potentially "more dangerous" case when compared to both the other cases, the text may be experienced also as more emotionally appealing and the communicative effect of the text may be enhanced by a reasoning process that also exploits aspects of imaginative selfidentification and/or empathy to promote vaccination [53]. Especially when compared to texts based on safe reasoning scenarios, the rebutting case is indeed highly visual (without having the contraindications of a real picture, [40]) and easier to emotionally share [13]. Previous literature also shows indeed that presenting factual descriptions are more successful communication strategies for vaccination commitment than presenting undercutting literal arguments against anti-vaccination myths $[27 ; 24]$. On the contrary, texts based on uncertain situations via undercutting defeaters are thought to increase the feeling of safety and the uptake of experts' advice at a collective level when compared to texts based on safe reasoning conditions. Differently from the reasoning conditions with rebutting defeaters, those with an undercutting defeater instead present a general condition that could undermine the conclusion, thus having stronger communicative effects at the collective level.

However, neither a main metaphorical framing effect nor a main defeasible reasoning effect was found on participants' acceptance of the conclusion, trust in institutions, and vaccination intentions (see Table 4). The metaphorical frame does not directly influence reasoning (as instead was found by Thibodeau and Borodisky $[66,67]$ ), but provides a more nuanced set of communicative effects. While metaphor was found to influence participants' 
acceptance of the conclusion in the case of syllogisms in narrow contexts [17], this effect seems lost in the case of wider contexts, where the persuasive effects of the text prevail over the argument evaluation. Also, metaphor use is not a predictor of higher vaccination intentions, as instead was found by Scherer and colleagues [54]. Presumably, just reading a text presenting a metaphor for vaccination is unlikely to directly produce a change in vaccination intentions, which are strongly linked to participants' previous beliefs on vaccination. As already discussed, the vaccination commitment and adherence depend on a more nuanced set of reasoning conditions, being also different at a personal and collective level. The results also disconfirm a main interaction between the metaphorical framing and the reasoning structure on the acceptability of the text conclusion and the overall set of communicative effects. However, even though the main effects of both the metaphorical frame and the defeasible reasoning on the acceptability of the conclusion are overall independent, the results partially confirm $\mathrm{H} 3$, showing a significant interaction effect in the case of the individual feeling of safety, trust in expertise and uptake of experts' advice, especially in the case of reasoning with undercutting defeaters.

The interaction is given by the significant difference between the metaphorical "undercutting" reasoning (MU) condition and the literal safe reasoning (LS) condition, as participants attributed higher value response to the former than the latter. When compared to the metaphorical safe reasoning (MS) condition, in (MU) condition participants attributed higher value responses also to the collective trust in institutions. Metaphor description of vaccination and defeasible reasoning support each other especially in (MU) condition, as the metaphor was extended in the text via the relevant property ("collaborative") underpinning a more general connection to the conclusion. The undercutting defeater would be the more relevant premise to invert the direction of the main inference to the conclusion, which is instead granted by both the conclusion of the text and the extended metaphor. Metaphors can indeed "influence the degree to which the recipient generates pro- or counter-arguments" [42, p. 787] and, especially when extended, metaphor can support the inference and the direction of elaboration of the argument where it appears. In this case, the metaphor - via its extended property - directs attention toward the most relevant feature from an argumentative point of view and supports the reasoning process, by activating an expectancy on the conclusion and making the overall text coherent. In directing attention to the most relevant property for the sake of argument, the metaphor can also have a corresponding directional effect on attitudes toward the topic of communication. Indeed, from a communicative perspective, this interaction between extended metaphor and reasoning with undercutting defeaters has the effect of making 
the interlocutor feel reassured by the expert and trust her expertise, possibly connected also to the increased uptake of experts' advice. The case of reasoning with rebutting defeaters is somewhat different under this respect, as it is based on a concrete case where the metaphorical relevant property is itself undermined (by the anti-vaccination collective behavior).

Previous studies supported the idea that metaphor can also influence people's attitudes and evaluation toward the communication source, thus affecting the credibility of the speaker and favoring attitude change [9]. The communicator credibility view suggests that communicators who use metaphors are judged more credible than ones who use literal language $[10,48]$. The reason is that novel metaphors may point out unexpected analogies, thus possibly leading to creative thinking and/or revision of (previous) untenable beliefs. As the success of health communication is intrinsically linked with patient trust [18; 71], metaphor use might turn out to be essential not only to the proper comprehension of vaccination, but also to therapy adherence [51]. However, previous studies provided mixed results on metaphor's enhancement of the source credibility $[60,48]$ and our study supports the idea that "well-used" metaphors in specific reasoning scenarios rather than metaphors tout court facilitate persuasion. As argued above, it is not the ability of highlighting relevant similarities between the beehive and vaccination per se that automatically makes the extended metaphor a good reasoning strategy for the overall argument. In the cases of vaccination we analyzed in this study, both a communicative and reasoning competence seems to be required to boost trust and vaccination commitment [71], which finally is at the core of the vaccine hesitancy problem $[33 ; 59]$.

Previous studies on the deliberate use of scientific metaphors in academic discourse [2] showed that there is an asymmetry in expertise between the expert and the addressee which might entail a mismatch in the uptake of the metaphor. Of course, also the listener's knowledge of the target domain is relevant, as it influences the path of possible sound inferences on the target issue. The mismatch might be simply due to the constitutive expert/laypeople asymmetry in terms of domain knowledge, which might be overcome by appropriate communicative/argumentative strategies [4]. However, pre-existing knowledge about the target [49] or strong ideological views about the target [32] could make the metaphor unlikely to change how people reason about the target domain, as well as their attitudes or behavior [42]. For instance, in the case of vaccination, Scherer and colleagues [54, p. 37] concluded that the effects of the metaphorical frames "were found among individuals who occasionally receive flu vaccinations but not among individuals who never or always receive flu vaccinations". Further research should be done on the influence of previous beliefs on individual differences in vaccination behavior: a trustful relationship with experts and institutions needs to be achieved 
even more so to counter vaccine hesitancy, which is not confined to people refusing (or encouraging others to refuse) vaccination [75].

\section{Conclusions}

The results of the study provided an overall picture of the communicative and reasoning competences far more nuanced than expected in using metaphor to promote vaccination. The study should be extended as it is limited for geographical area, and also presented in another time-framework, when not (directly) influenced by the quite recent COVID-19 outbreak. Further research should also consider conventional and non-extended metaphors as well as emotive metaphors (i.e. metaphors having positive/negative valence) to compare the metaphorical communicative effects, especially in terms of emotional impact and persuasion, on vaccination uptake. Also, alternative reasoning scenarios should be considered, to further understand the different role of rebutting vs. undercutting defeaters in reasoning about vaccination.

Metaphor does not automatically increase vaccination commitment and trust. Not even defeasible reasoning, as a specific competence of experts', can do that. Rather, both a communicative and defeasible reasoning competence is needed to enhance trust on immunization, with possible different outcomes at an individual and collective level. As in other fields of science communication in general and health communication in particular, metaphor can be an opportunity for a good argument, constructing social bonding but also committing to both the risks and responsibilities of communication [19]. Depending on the argumentative structure and the kind of defeasible envisaged alternatives, metaphor can also present an imagined scenario (with its possible features and consequences) we might or not adhere to [16], influencing our uptake of the overall argument.

\section{Acknowledgements}

The authors thank all the participants to the study, which is part of a wider ongoing project "Metaphors for Vaccination and Defeasible Reasoning". World Pandemic Research Network. WPRN-439252: https://wprn.org/item/439252. 


\section{References}

1. Baake K. Metaphor and knowledge: The challenges of writing science. New York: Suny Press; 2003.

2. Beger A. The contested notion of 'deliberate metaphor': What can we learn from 'unclear' cases in academic lectures? Yearb Ger Cogn Linguist Assoc. 2019; 7(1): 5166.

3. Biasio L, Corsello G, Costantino C, Fara GM, Gianmanco G, Signorelli C, Vecchio D, Vitale F. Communication about vaccination: A shared responsibility. Hum Vaccin Immunother. 2016; 12(11): 2984-2987.

4. Bigi S. Communicating (with) care. Amsterdam: IOS Press; 2016.

5. Biss E. On immunity: An inoculation. Minneapolis: Graywolf Press, 2014.

6. Black M. Models and metaphors: Studies in language and philosophy. Ithaca: Cornell University Press; 1962.

7. Bouder F. Risk Communication of vaccines: Challenges in the post-trust environment. Current Drug Safety. 2015; 10: 9-15.

8. Bowdle B, Gentner D. The career of metaphor. Psychol Rev. 2005; 112(1): 193-216.

9. Bowers JW, Osborn MM. Attitudinal effects of selected types of concluding metaphors in persuasive speeches. Speech Monographs. 1966; 33(2): 147-155.

10. Brugman BC, Burgers C, Vis B. Metaphorical framing in political discourse through words vs. concepts: A meta-analysis. Lang Cogn. 2019; 11(1): 41-65.

11. Charteris-Black J. Forensic deliberations on 'purposeful metaphor'. Metaphor Soc World. 2012; 2(1): 1-21.

12. Deignan A. Deliberateness is not unique to metaphor: A response to Gibbs. Metaphor Soc World. 2011; 1(1): 57-60.

13. Dubé E, Gagnon D, Vivion M. Optimizing communication material to address vaccine hesitancy. Can Commun Dis Rep. 2020 Feb 6;46(2-3):48-52. doi: 10.14745/ccdr.v46i23a05.

14. Ervas F, Gola E, Rossi MG. Metaphors and emotions as framing strategies in argumentation. In: Airenti G, Bara B, Sandini G, editors. EAP CogSci. Proceedings of the EuroAsianPacific Joint Conference on Cognitive Science. vol. 1419 of Torino: CEUR Workshop Proceedings; 2015. pp. 645-650.

15. Ervas F. From the "Garrison" to the "Beehive". Metaphors and framing strategies in vaccine communication. Politeia. 2018; 130: 28-37. 
16. Ervas F. Metaphor, ignorance, and the sentiment of (ir)rationality. Synthese. 2019 Nov 28. doi: 10.1007/s11229-019-02489-y.

17. Ervas F, Ledda A, Ojha A, Pierro GA, Indurkhya B. Creative argumentation: When and why people commit the metaphoric fallacy. Front Psychol. 2018 Sep 25; 9:1815. doi: 10.3389/fpsyg.2018.01815.

18. Fallowfield L, Jenkins V. Effective communication skills are the key to good cancer care. Eur J Cancer. 1999; 35(11): 1592-1597.

19. Frezza G. Metaphor: the good argument in science communication. RIFL 2016; 10(2): 21-33.

20. Gibbs RW. Are 'deliberate' metaphors really deliberate? a question of human consciousness and action. Metaphor Soc World. 2011; 1(1): 26-52.

21. Gibbs RW. Does deliberate metaphor theory have a future? J Prag. 2015; 90: 73-76.

22. Gick ML, Holyoak KJ. The cognitive basis of knowledge transfer. In: Cormier SM, Hagman JD, editors. The educational technology series. Transfer of learning: Contemporary research and applications. San Diego, CA: Academic Press; 1987. pp. 946.

23. Greenberg J, Dubé E, Driedger M. Vaccine hesitancy: In search of the risk communication comfort zone. PLoS Curr. 2017 Mar 3; 3;9: ecurrents.outbreaks.0561a011117a1d1f9596e24949e8690b.

24. Grignolio A. Vaccines: Are they worth a shot? Cham: Springer; 2018.

25. Hauser DJ, Schwarz N. The war on prevention: Bellicose cancer metaphors hurt (some) prevention intentions. Pers Soc Psychol Bull. 2015; 41(1): 66-77.

26. Hesse M. Theories, Family resemblances and analogy. In: Helman DH, editor. Analogical reasoning. Synthese Library (Studies in Epistemology, Logic, Methodology, and Philosophy of Science). Dordrecht: Springer; 1988. pp. 317-340.

27. Horne Z, Powell D, Hummel JE, Holyoak KJ. Countering antivaccination attitudes. PNAS 2015 Aug 3; 112(33): 10321-10324. doi: 10.1073/pnas.1504019112.

28. Keefer LA, Landau MJ, Sullivan D, Rothschild ZK. Embodied metaphor and abstract problem solving: Testing a metaphoric fit hypothesis in the health domain. J Exp Soc Psychol. 2014; 55: 12-20.

29. Kelly T. Evidence. In: Zalta EN, editor. The Stanford Encyclopedia of Philosophy. 2014; plato.stanford.edu/entries/evidence

30. Lakoff G, Johnson M. Metaphors we live by. Chicago: Chicago University Press; 1980. 
31. Lakoff G. Don't think of an elephant: Know your values and frame the debate. London: Chelsea Green Publishing; 2004.

32. Landau MJ, Keefer LA, Rothschild ZK. Epistemic motives moderate the effect of metaphoric framing on attitudes. J Exp Soc Psychol. 2014; 53: 125-138.

33. Larson HJ, Clarke RM, Jarrett C, Eckersberger E, Levine Z, Schulz WS, Paterson P. Measuring trust in vaccination: A systematic review. Hum Vaccin Immunother. 2018; 14(7): 1599-1609.

34. Macagno F, Rossi MG. Metaphors and problematic understanding in chronic care communication. J Prag. 2019; 151: 103-117.

35. MacDonald NE. Vaccine hesitancy: Definition, scope and determinants. Vaccine. 2015; 33(34): 4161-14164.

36. Mayer RE. Thinking, problem solving, cognition. New York: Freeman; 1992.

37. Müller C. Are 'deliberate' metaphors really special? Deliberateness in the light of metaphor activation. Metaphor Soc World. 2011; 1(1): 61-66.

38. Navin M. Values and vaccine refusal: Hard questions in ethics, epistemology, and health care. New York: Routledge; 2015.

39. Novick LR. Representational transfer in problem solving. Psychol Sci. 1990; 1(2): 128132.

40. Nyhan B, Reifler J, Richey S, Freed GL. Effective messages in vaccine promotion: A randomized trial. Pediatrics 2014; 133(4) e835-e842. doi: 10.1542/peds.2013-2365.

41. Oswald S, Rihs A. Metaphor as argument: Rhetorical and epistemic advantages of extended metaphors. Argumentation 2014; 28(2):133-159.

42. Ottati VC, Renstrom RA. Metaphor and persuasive communication: A multifunctional approach. Soc. Personal. Psychol. Compass. 2010; 4(9): 783-794.

43. Peretti-Watel P, Verger P, Raude J, Constant A, Gautier A, Jestin C, Beck F. Dramatic change in public attitudes towards vaccination during the 2009 influenza $A(H 1 N 1)$ pandemic in France. Euro Surveill. 2013 Oct 31;18(44):20623. doi: 10.2807/15607917.es2013.18.44.20623.

44. Pollock J. The structure of epistemic justification. Am Philos Q 1970; 4: 62-78.

45. Pollock J. Knowledge and justification. Princeton: Princeton University Press; 1974.

46. Reed SK. Word problems. Mahwah, NJ: Lawrence Erlbaum Associates, Inc.; 1999.

47. Reintjes R, Das E, Klemm C, Richardus JH, Keßler V, Ahmad A. "Pandemic public health paradox": Time series analysis of the 2009/10 Influenza A / H1N1 Epidemiology, 
Media Attention, Risk Perception and Public Reactions in 5 European Countries. PLoS ONE 2016; 11(3): e0151258. doi: 10.1371/ journal.pone.0151258

48. Reinsch NL. Figurative language and source credibility: A preliminary investigation and reconceptualization. Hum Commun Res. 1974; 1: 75-80.

49. Reuchamps M, Dodeigne J, Perrez J. Changing your political mind: The impact of a metaphor on citizens' representations and preferences for federalism. Regional \& Federal Studies. 2018; 28(2): 151-175.

50. Robins S, Mayer RE. The metaphor framing effect: Metaphorical reasoning about textbased dilemmas. Discourse Processes. 2000; 30(1): 57-86.

51. Rossi MG, Macagno F. Coding problematic understanding in patient-provider interactions. Health Commun. 2020; 35 (12): 1487-1496.

52. Salis P, Ervas F. Evidence, defeasibility, and metaphors in diagnosis and diagnosis communication. Topoi. 2020. doi: 10.1007/s11245-020-09698-y

53. Shelby A, Ernst K. Story and science: How providers and parents can utilize storytelling to combat anti-vaccine misinformation. Hum Vaccin Immunother. 2013; 9(8): 17951801 .

54. Scherer AM, Scherer LD, Fagerlin A. Getting ahead of illness: Using metaphors to influence medical decision making. Medical Decision Making. 2015; 35(1): 37-45.

55. Semino E. Metaphor in discourse. Cambridge: Cambridge University Press; 2008.

56. Semino E, Demjen Z, Demmen J. An integrated approach to metaphor and framing in cognition, discourse and practice. Appl Linguist 2016; 5: 1-22.

57. Semino E, Demjén Z, Hardie A, Payne S, Rayson P. Metaphor, cancer and the end of life. Routledge, New York; 2018.

58. Skelton J, Wearn AM, Hobbs R. A concordance-based study of metaphoric expressions used by general practitioners and patients in consultation. Br J Gen Pract. 2002; 52(475): 114-118.

59. Sondagar C, Xu, R, MacDonald NE, Dubé E. Vaccine acceptance: How to build and maintain trust in immunization. Can Commun Dis Rep. 2020; 46(5): 155-159.

60. Sopory P, Dillard J. The persuasive effects of metaphor. Hum Commun Res 2002; 28: 382-419.

61. Sontag S. Illness as metaphor. New York: Farrar, Straus \& Giroux; 1978.

62. Steen GJ. The paradox of metaphor: Why we need a three-dimensional model of metaphor. Metaphor Symb 2008; 23: 213-241. 
63. Steen GJ. The contemporary theory of metaphor - Now new and improved! Review of Cogn Linguist. 2011; 9: 26-64.

64. Steen GJ, Reijnierse G, Burgers C. When do natural language metaphors influence reasoning? A follow-up study to Thibodeau and Boroditsky (2013). PLoS ONE 2014; 9(12): e113536. doi: 10.1371/journal.pone.0113536.

65. Steen GJ. Deliberate metaphor theory: Basic assumptions, main tenets, urgent issues. Intercult Pragmat 2017; 14(1): 1-24.

66. Thibodeau PH, Boroditsky L. Metaphors we think with: The role of metaphor in reasoning. PLoS ONE 2011; 6(2): e16782. doi: 10.1371/journal.pone.0016782.

67. Thibodeau PH, Boroditsky L. Natural language metaphors covertly influence reasoning. PLoS ONE 2013; 8(1): e52961. doi: 10.1371/journal.pone.0052961.

68. Thibodeau PH, Hendricks RK, Boroditsky L. How linguistic metaphor scaffolds reasoning. Trends Cogn Sci. 2017; 21(11): 852-863.

69. Thibodeau PH, Matlock T, Flusberg SJ. The role of metaphor in communication and thought. Lang Linguist Compass. 2019 May 9; e12327. doi: 10.1111/lnc3.12327.

70. Thibodeau PH. Extended metaphors are the home run of persuasion: Don't fumble the phrase. Metaphor Symb. 2016; 31: 53-72.

71. Thom DH. Physician behaviors that predict patient trust. J Fam Pract. 2001; 50(4): 323328.

72. Tversky A, Kahneman D. Extensional versus intuitive reasoning: The conjunction fallacy in probability judgment. Psychol Rev. 1983; 90(4): 293-315.

73. van Poppel L. The study of metaphor in argumentation theory. Argumentation. 2020; doi: 10.1007/s10503-020-09523-1.

74. van Poppel L. The relevance of metaphor in argumentation. Uniting pragma-dialectics and deliberate metaphor theory. J Prag. 2020; 170: 245-252.

75. Yaquib O, Castle-Clarke S, Sevdalis N, Chataway J. Attitudes to vaccination: A critical review. Soc. Sci. Med. 2014; 112: 1-11.

\section{Supporting information}

S1 File. Supplementary material. Table 1. Argumentative texts on vaccination in Italian and Table 2. Questionnaire in Italian and translation into English. 